\title{
Detection of Magnetic Fields Created by Biological Tissues
}

\section{Ranjith S. Wijesinghe*}

Department of Physics and Astronomy, Ball State University, Muncie, USA

\begin{abstract}
The recording of magnetic fields associated with cellular current flow in nerve and muscle fibers was first reported nearly three and a half decades ago. These magnetic fields are detected using a room-temperature toroidal pickup coil called the neuromagnetic current probe. These probes can be employed to measure action currents of biological tissues while they are resting in their own natural settings or in saline baths, thereby reducing the risk associated with elevating and drying the tissues in the air during experiments. These magnetic fields are relatively insensitive to muscle movement since these probes are not directly connected to the tissues, and distortions of the recorded data due to changes in the electrochemical interface between the probes and the tissues are minimal. In this paper, the magnetic fields of single nerve axons, muscle fibers and nerve bundles are discussed. Furthermore, one of the possible applications of the neuromagnetic current probe to the intraoperative assessment of damaged nerve bundles is discussed.
\end{abstract}

Keywords: Neuromagnetic current probe; Nerve; Muscle; Nerve conduction velocity distributions

\section{Background}

The original measurements of human Magnetocardiogram (MCG), the Magnetoencephalogram (MEG) and the Magnetomyogram clearly demonstrated the existence of the magnetic fields that are associated with ionic action currents in electrically active biological tissues [1$3]$. Because of the heart can produce a relatively large magnetic field compared with the brain and some other organs, the early research on biomagnetic fields originated with mathematical modeling of MCG. The early experimental studies involving animal studies were also concentrated largely on the MCG. In addition, those experimental data suffered from poor spatial resolution and low sensitivity because of the lack of sophisticated detecting instruments. With improved technologies, the investigations expanded into brain function and preliminary studies of the evoked MEG started to emerge in the 1980s. Those studies provided some details about which neuronal populations were contributing to the magnetic signals arising from the brain. However, the signals from single neurons were too weak to be detected, and a group of more than 10000 dendrites were needed as a group to detect MEGs [4]. At the time, abundant physiological, technical, and mathematical limitations hampered quantitative comparisons of theory and experiments involving human ECGs as well as other biomagnetic recordings. The lack of accurate microscopic source models made it even harder to agree upon what specific physiological factors affected the strength of the MEG and other biomagnetic signals, and which factors dominated the attainable spatial resolutions [4].

Over the last three and a half decades, a significant number of studies have been conducted to measure and analyze the magnetic signals created by ionic currents flowing in isolated nerve axons and muscle fibers. These measurements have been supported by theoretical investigations and development of a room-temperature amplifier and a neuromagnetic current probe. Now magnetic recording at the cellular level has been well established as a quantitative measurement technique of action current. This paper first describes the generation of cellular magnetic field and the instrumentation necessary to detect these signals. It then presents the very first investigations on magnetic fields created by single axons and bundles. It finally discusses non-uniform propagation of action signals, and some possible clinical applications of this technique to investigate injured nerve bundles.

\section{Magnetic Fields Created by Nerve Axons and Muscle Fibers}

Electrically active nerve and muscle fibers create propagating action potentials and magnetic fields. Therefore both the electrical potential and the magnetic field provide very valuable source of information about the properties of nerve and muscle fibers. The typical strength of the encircling magnetic field is indicated with the wide arrows in Figure 1. According to Ampere's law, the strength of this field is proportional to the total current flowing through the neuromagnetic current probe.

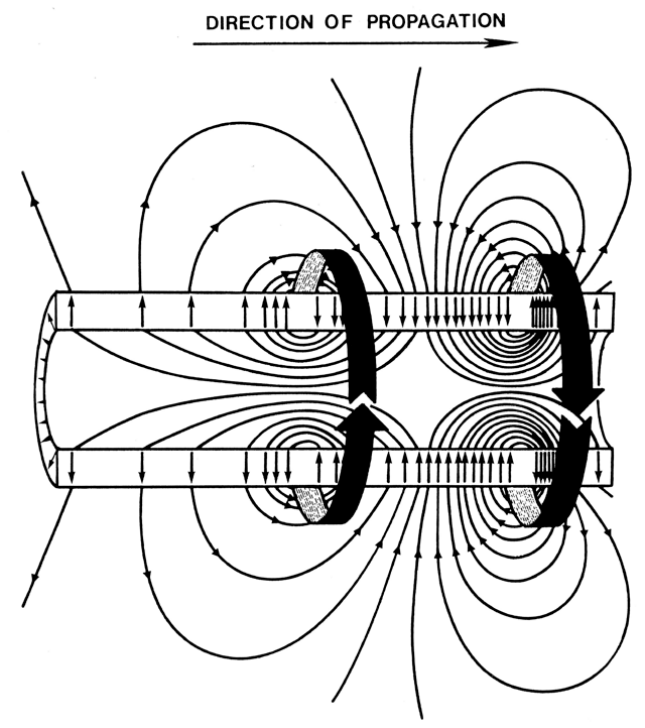

Figure 1: A cross-section of a nerve axon showing the electric current (thin lines) and the magnetic field (wide bands) associated with a propagating nerve action signal. The membrane thickness is greatly exaggerated (Reproduced from Wikswo [53]).

*Corresponding author: Ranjith S. Wijesinghe, Department of Physics and Astronomy, Ball State University, Muncie, IN 47306, USA, Tel: 765-285-8811; Fax: 765-285-5674; E-mail: rswijesinghe@bsu.edu

Received January 15, 2014; Accepted February 03, 2014; Published February 05, 2014

Citation: Wijesinghe RS (2014) Detection of Magnetic Fields Created by Biological Tissues. J Electr Electron Syst 3: 120. doi:10.4172/2332-0796.1000120

Copyright: $\odot 2014$ Wijesinghe RS. This is an open-access article distributed under the terms of the Creative Commons Attribution License, which permits unrestricted use, distribution, and reproduction in any medium, provided the original author and source are credited. 
The typical strength of the encircling magnetic field at $1 \mathrm{~mm}$ from a 10 $\mu \mathrm{m}$ diameter nerve axon is about 50 femto Tesla [4].

The earliest attempt to measure the magnetic field of an isolated biological tissue was made more than three and a half decades ago. The first successful measurement of the magnetic field produced by a peripheral nerve was reported in 1980 [5] using a frog sciatic nerve bundle. Since then, the same research group has been investigating the theoretical aspects, the instrumentation requirements, and the experimental techniques required for both the basic and the applied research into cellular magnetic fields. The original toroidal pickup coil was built using a ferrite-core with $1.2 \mathrm{~mm}$ width, $0.6 \mathrm{~mm}$ inner radius, and $1.3 \mathrm{~mm}$ outer radius, and it was wound with just four turns of copper wire. They immersed both the toroidal pickup coil and the nerve in a saline bath and used a very brief electric pulse to stimulate the nerve. They had designed the pickup coil so that it was able to block the return current through the pickup coil. A Superconducting Quantum Interference Device (SQUID) magnetometer detected the current induced in the toroid winding, due to the ionic or action currents in the nerve bundle. The first peak of the observed biphasic magnetic signal had $70 \mathrm{pT}$ of amplitude with $1 \mathrm{msec}$ total duration. It was also noticed that a reversal of polarity when the direction of the propagation was reversed. These investigators have subsequently improved their recording techniques and instrumentation [6-9] to a point where a variety of isolated, one-dimensional tissues can be studied [10-13]. One of the key benefits of developing the room-temperature amplifier was that it was sensitive enough to measure the current in the toroidal pickup coil windings, thereby eliminating the cost and inconvenience of cryogenic technology $[14,15]$.

Later the same research group made the simultaneous measurements of the magnetic field and transmembrane potential created by just a single axon [16-18] using the very same technique employed during their previous experiments. A schematic diagram of their experiment setup is shown in Figure 2. The measured magnetic field and the transmembrane potential of the crayfish giant axon are shown in Figure 3. These data sets were analyzed using their own mathematical models

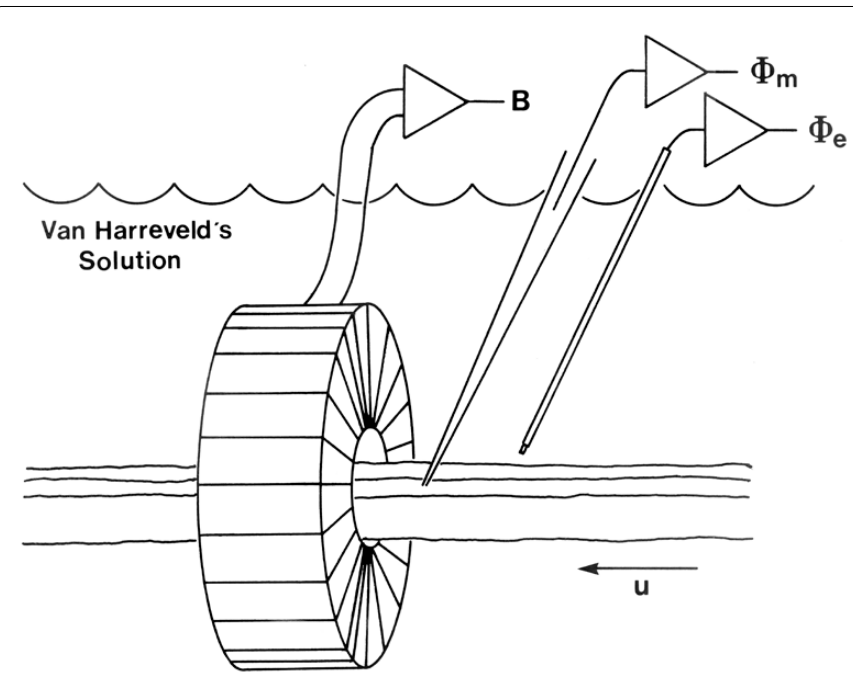

Figure 2: Schematic diagram of an experiment to measure the transmembrane potential $\Phi_{m}$ the extracellular potential $\Phi$, and magnetic field B produced by a single crayfish giant axon. A toroid is used to measure the magnetic field, a glass microelectrode is used to measure the transmembrane potential, and an extracellular electrode is used to measure the extracellular action potential (Reproduced from Roth and Wikswo [17]). a)

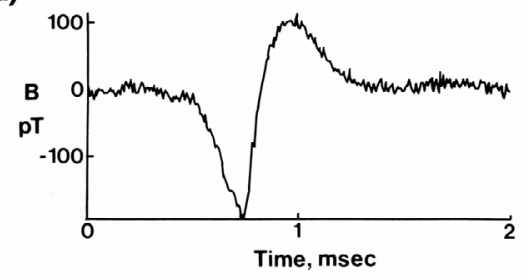

b)

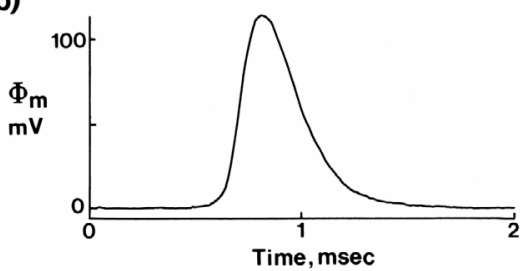

Figure 3: (a) An un-averaged magnetic field $B$ and (b) the transmembrane potential $\Phi_{m}$ measured from an isolated crayfish giant axon (Reproduced from Roth and Wikswo [17]).

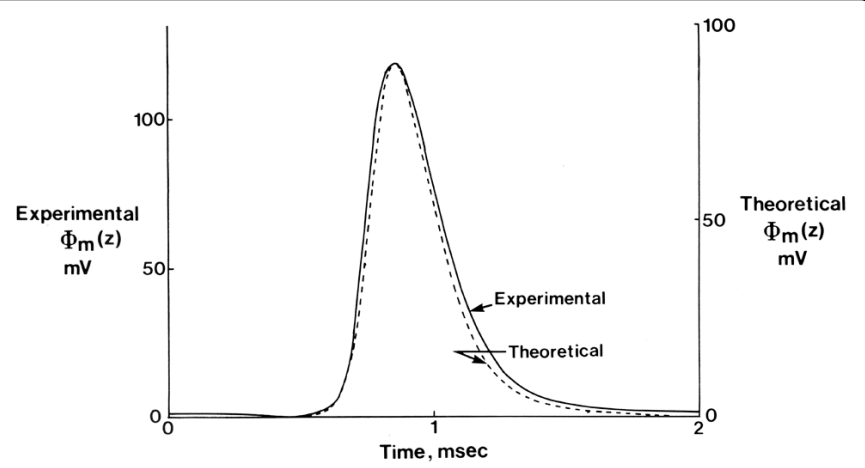

Figure 4: Comparison of the measured transmembrane potential (512 averages) and the transmembrane potential calculated from measured magnetic field. (Reproduced from Roth and Wikswo [17]).

which had been developed specifically for analyzing the magnetic field produced by nerve axons [19-24]. As noted before, by Ampere's law, the magnetic field measured by the toroid is proportional to the net current flowing through it. However, the net current is the sum of the intercellular current and fraction of the extracellular current passing through the neuromagnetic current probe called the return current. For a probe with a small inner radius, the return current is negligible and the recorded magnetic signal is due mainly to the intracellular current, which is equal to the axial derivative of the transmembrane potential divided by the resistance per unit length of the fiber [23]. If the probe is large enough that the return current is significant, it cannot be neglected. In that case, the return current can be evaluated using Ohm's law once the extracellular potential of the tissue sample is known. The extracellular potential can be obtained by solving the Laplace equation [24,25]. Later the inverse problem associated with recorded single axon magnetic fields, which is the calculation of the transmembrane potential from the measured magnetic field, was also investigated [24]. Figure 4 shows a comparison of the measured transmembrane potential and the calculated transmembrane potential from the measured magnetic field of an isolated crayfish giant axon. While the shapes of these two traces agree quite well, their amplitudes do not. However, these investigators found that they could adjust just one unknown parameter in the model until the amplitudes of those two 


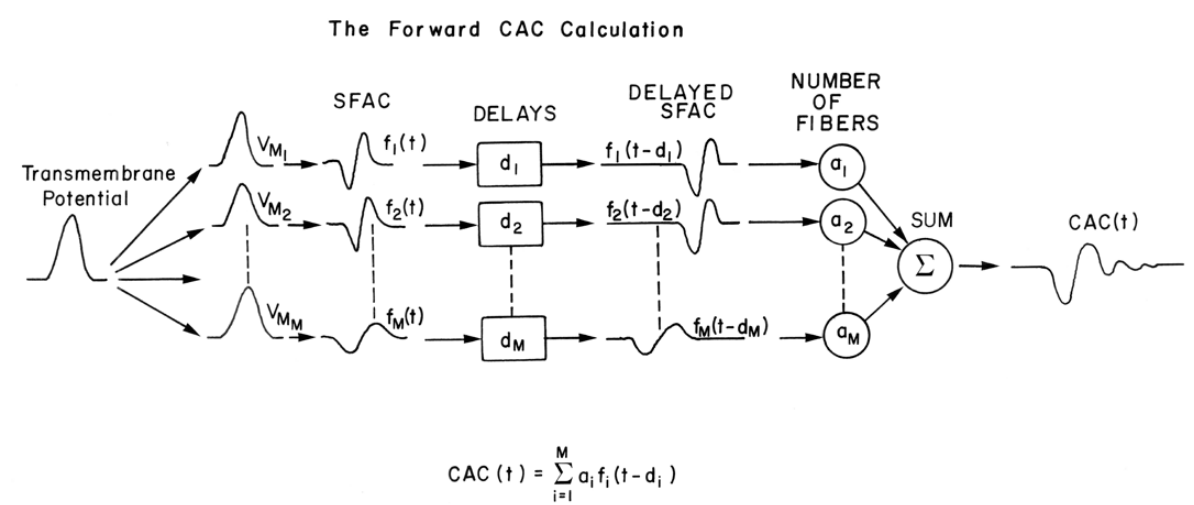

Figure 5: Block diagram of the forward procedure for simulating the Compound Action Current (CAC). The Single Fiber Action Currents (SFACs) in each of M conduction velocity classes are calculated from an assumed transmembrane potential using a generalized volume conduction model of an axon in a nerve bundle. Then the SFACs are delayed according to their conduction velocity and the distance between the stimulus and recording site, are multiplied by the number of fibers in each class, and are summed to obtain the CAC (Repoduced from Wijesinghe, et al. [25]).

a)

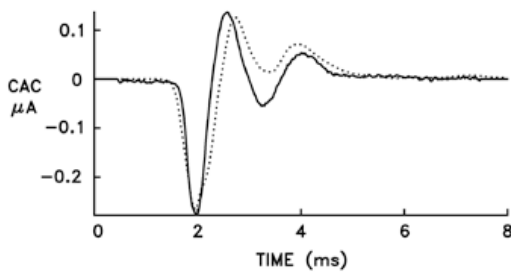

c)
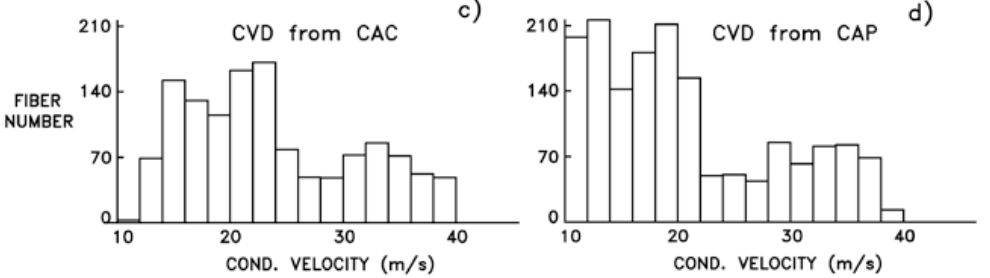

b)

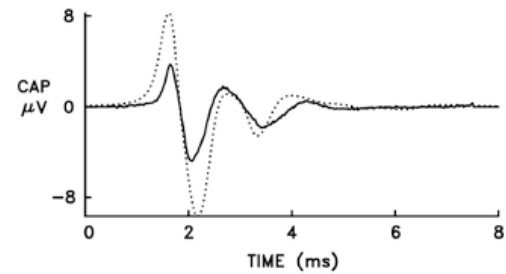

d)

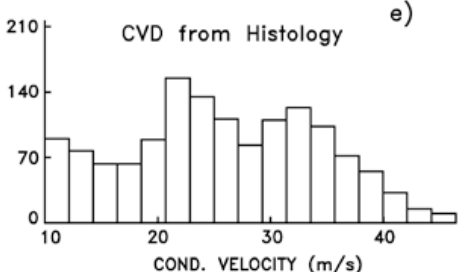

Figure 6: The a) Compound Action Current (CAC), and b) Compound Action Potential (CAP) recorded from a bull frog sciatic nerve bundle at $21^{\circ} \mathrm{C}$. The propagation distances for the CAC and CAP were $78 \mathrm{~mm}$ and $60 \mathrm{~mm}$, respectively. The Conduction Velocity Distribution (CVD) predicted from the c) CAC and d) $\mathrm{CAP}$, and e) the histologically measured CVD (Reproduced from Wijesinghe, et al. [27]).

traces matched. Using this technique for the intracellular conductivity, they found the intracellular conductivity of a crayfish giant axon was $1.44 \mathrm{~S} / \mathrm{m}$ with $\pm 0.33 \mathrm{~S} / \mathrm{m}$ error.

The first successful measurement of compound action current associated with a nerve bundle using the magnetic technique was reported by the same research group in 1991 [26,27]. Performing an experiment on an isolated frog sciatic nerve bundle with the neuromagnetic current probe, they measured the compound action current associated with the individual ionic current in each axon in the nerve bundle. During the same experiment, they simultaneously recorded the compound action potentials created by each individual axon in the bundle. These investigators concurrently kept developing the mathematical models needed for analyzing the recorded data $[25,26]$. In doing so, they used a generalized volume conduction model to simulate the single fiber action signals needed for the inverse model [25]. Frequency dependent conductivities, variations in the intracellular action potentials with recording temperature and axon conduction velocity, and the effect of axonal myelination were incorporated into the volume conduction calculation. Figure 5 depicts a block diagram of their forward calculation which simulated the compound action signals [26]. Using recorded magnetic and electric signals as inputs for their inverse model [26], they calculated the fiber diameter histograms of the nerve bundle and compared them with the fiber diameter histogram which was obtained from the histologically prepared nerve bundle after the experiment. Figure 6 shows the recorded signals during one of the experiments and the corresponding Conduction Velocity Distribution (CVD) histograms. The agreement between the three predicted CVDs is best for the faster conduction velocity classes, whereas the slower classes are more susceptible to errors primarily due to the recording noise embedded in the data. This detailed analysis showed that the magnetic technique may have some advantages over that of electric technique in determining the fiber diameter histograms. In addition, the same research group investigated the effect of recording temperature on the histograms and the conduction velocity of individual action signals.

Action signal propagation that exhibits a variation in the shape of signals along the sample (non-uniform propagation) is difficult 
to measure using the traditional electric methods because it requires either multiple simultaneous measurements, or sequential recordings made while scanning the sample. The traditional intracellular recording methods cannot be applied for this purpose because of the risk of cell damage inherent from multiple electrode impalements. Also extracellular electric potential measurements do not provide accurate quantitative information [27]. However, the neuromagnetic current probe takes advantage of the absence of physical contact between the probe and the sample and allows these measurements to be made on isolated nerve axons. Exploiting this advantage, Van Egeraat et al. [28] recorded the response of a crayfish medial giant axon to a nerve crush with the help of a neuromagnetic current probe. The experimental data that they recorded were interpreted with a mathematical model that incorporated both the radial and axial ionic transport and membrane kinetics. Their experiments showed that the effects of the crush were manifested statically as an increase of the resting potential and dynamically as a reduction in the amplitude of the action current and potential, and were observable as far as $10 \mathrm{~mm}$ from the crush site. In addition, the normally biphasic magnetic signal became monophasic near the crush. Their mathematical model reflected these observations accurately. Based on the experimental data, their mathematical model predicted that the crush seals with a time constant of $45 \mathrm{~s}$. They also estimated the injury current density entering the axon through the crush to be initially on the order of $0.1 \mathrm{~mA} / \mathrm{mm}^{2}$. That current lasted until the crush sealed or the concentration gradients between the intra- and extracellular spaces came to equilibrium. Later these investigators [29] developed an analytical model to investigate the axonal propagation incorporating both the radial and axial transport in an axon. The main goal of that investigation was to understand the long-term behavior of transected or injured axons.

Taking their research effort to another level which was facilitated by the neuromagnetic current probe, Egeraat, et al. [30] reported the first measurement of the magnetic field recorded from a single muscle fiber. They reported that the current associated with the magnetic field of a single muscle fiber was also biphasic and had peak-to-peak amplitudes ranging between 50 and $100 \mathrm{nA}$. These amplitudes mostly depended upon the fiber diameter. Following the same experimental technique which was employed on the crayfish giant axon and using a core conductor model, they calculated the transmembrane potential of the muscle fiber using the recorded magnetic field. Their estimated values for the intracellular conductivity and the effective membrane capacitance of the muscle fiber were $0.20 \pm 0.09 \mathrm{~S} / \mathrm{m}$ and $0.030 \pm$ $0.011 \mathrm{~F} / \mathrm{m}^{2}$, respectively. They also demonstrated that the anisotropic conductivity of the muscle bundle did not significantly affect these estimated values.

\section{Role of Magnetic Technique in Clinical Investigations}

Kline and his colleagues first introduced electrophysiologic methods to clinical settings hoping to provide both qualitative and quantitative determinations of the permanence of the axons at a site of nerve injury [31-34]. They performed intraoperative electrophysiologic assessments of nerve bundles by stimulating and recording electric signals (compound action potentials) from them. Six years later, using the then-latest developments in microsurgical techniques, Terzis et al. [35] refined intraoperative assessment by stimulating and recording electric signals from individual fascicles or groups of fascicles. These recording techniques have all utilized a pair of closely separated electrodes that raise the nerve bundle into the air from its original position, which could lead to drying up the preparation unless it is continuously kept moisturized. In addition, reliable and repeatable measurements of amplitude, conduction velocity, and shape of the recorded electric signal require very careful and reproducible electrode placement $[36,37]$. This may also require significant dissection of the nerve and keeping the nerve suspended in the air for a considerable period, exposing the nerve to the danger of cell death. In addition, small changes in electrode placement or the conductivity of the layer of moisture on the nerve makes it difficult to obtain reproducible data [31]. The magnetic technique is therefore ideally suited to overcome the deficiencies of the traditional electric technique.

The instrumentation and mathematical models developed for the purpose of studying the magnetic field created by an isolated single axon can be likewise applied to clinical research with some modifications. For this purpose, a crude openable neuromagnetic current probe was developed [31] with the intention of showing that the magnetic fields created by intact nerve bundles could be measured. After the nerve has been surgically exposed, the intact nerve can be enclosed by the openable probe. Then it can be stimulated proximal to the injury, and the probe can be scanned over the nerve bundle to record action signals along the bundle. Vanderbilt research group achieved the first and only reported intraoperative recordings of the Compound Action Current (CAC) from the human median nerve in the literature [38], from a patient undergoing surgical section of the flexor retinaculum for decompression of the carpal tunnel. They exposed the median nerve proximal to the flexor retinaculum and followed distally to demonstrate the lateral and medial branches. A $2 \mathrm{~mA}$ amplitude pulse with $100-\mu_{\mathrm{s}}$ duration was used for the nerve stimulation. The openable neuromagnetic current probe was placed in the palm of the hand to encircle the primary branch of the median nerve innervating the index and middle fingers, $6.0 \mathrm{~cm}$ distal to the stimulus electrodes. The nerve response was extracted and the peak-to-peak amplitude of the signal was about $0.65 \mu \mathrm{A}$. Using their inverse mathematical model $[26,27]$, these investigators estimated the Conduction Velocity Distribution (CVD) of the nerve bundle. Similar to previous observations of animal preparations, the slower conduction velocity classes have been over estimated in the CVD mainly due to the recorded noise embedded in the signal and uncertainties in the model parameters. Therefore, in general, the magnetic technique offers the advantages of being made with the nerve immersed in saline, and it appears that magnetic technique is easier to utilize in clinical settings than the electric technique. Furthermore, magnetic measurements are more accurate, less noisy, and less vulnerable to artifact than those of the traditional electric measurements [39].

The neuromagnetic current probe is a valuable research tool for the biomedical research community. However, its research capabilities have not yet been fully exploited. A more recent publication [40] concludes, based on the previous findings involving the neuromagnetic current probe, that the currently available MRI procedures may not be sufficient to detect the neuronal currents in peripheral nerves and muscles. This issue has been the main focus of more than a dozen recently published papers [41-45] even though the data required to come to the above conclusion was already in the literature $[40,46,47]$.

\section{Magnetic Technique and Nerve Regeneration}

The quality of a peripheral nerve reconstruction is primarily evaluated based on the functional recovery. In most cases, a connective scar tissue is formed around a nerve bundle following an injury to a peripheral nerve bundle thus preventing proper regeneration of the nerve axons, and surgical procedures must be explored to restore the nerve function. Results are generally unsatisfactory due to the formation of scars [39]. To investigate this important surgical question, action 
signal propagation along nerve graft in a monkey has been studied [39] using the neuromagnetic current probe. The median nerve of a Macaca fascicularis primate was exposed at the wrist and a $15-\mathrm{cm}$ long segment was excised. Then the nerve was incised and sutured together. Six months were allowed for the nerve axons to regenerate across the repair site and into the hand, after which the median nerve was exposed surgically once more and the probe was placed around the nerve at the elbow. Then the nerve was stimulated at both the proximal and distal to the grafted segment. The difference in amplitude of the signals displays the amount of the axons in the nerve that have effectively linked both sides of the graft. The calculated conduction velocities of these two signals indicate that the conduction velocity is also decreased in the regenerated part of the nerve. In 1993, Kuypers et al. [48] further investigated the merits of nerve compound action signals recorded using the electric and magnetic techniques. They compared the two techniques using the rabbit peroneal nerve after a nerve reconstruction. They recorded signals $2,4,6$, and 8 weeks after the nerve reconstruction and also concluded that magnetic signals were far more reproducible and less prone to artifacts than electric signals. Kuypers' group also reported that the magnetic recording was able to demonstrate that the number of axons that had regenerated increased with time, which could not be achieved using the traditional electric recordings or histological methods. These investigators also found another unique feature coupled with the magnetic technique. They quantified the number of functional myelinated neuronal units in the peroneal nerve of New Zealand White rabbits 20 weeks after the reconstruction. They found that the magnetic technique was the only technique that could quantitatively predict how many regenerated fibers in a nerve bundle could conduct action signals after a reconstruction [49]. The reason behind this finding was that the even though histologic techniques could quantify the number of axons in a nerve, they were unable to provide information about their ability to conduct an action signal. There was a significant difference in conductive and nonconductive axons in reconstructed nerves. Another important observation which Kuypers and colleagues made was that the magnetic technique could be useful in evaluating the success of peripheral nerve reconstruction shortly after it was performed [50]. The magnetic technique appeared to be capable of predicting the eventual functional recovery of the nerve, based on the quality of the amplitude of the signal recorded during the early stages of recovery. This outcome is very beneficial for neurosurgeons. These investigations indicate the feasibility of magnetic measurement of nerve regeneration in mammals.

\section{Limitations of Magnetic Technique}

The magnetic measurement technique has several remarkable advantages. In most cases, as described in this paper, a single magnetic measurement is adequate to decide quantitatively what is the intracellular action current of an axon without making assumptions regarding tissue resistivity. This has not been the case with the traditional electric methods. On the other hand, magnetic and electric measurements can be pooled to obtain new information on tissue parameters such as intracellular conductivity [17] and anisotropy [51]. There is no need for physical contact between the probe and the tissue sample during the recording of magnetic signals. Therefore, the sample can be scanned along its axial direction to obtain the spatial variation in the signals. Electric measurements with intracellular microelectrodes would necessitate multiple electrode insertions with great risk of damage to the sample. Because of this advantage associated with magnetic technique, magnetic measurements create the opportunity for researchers to study non-uniform propagation in systems with an axial inhomogeneity, such as a nerve crush, bifurcations, synapse, and tapered fibers or bundles. Although extracellular electric measurements can also be used for scanning, the quantitative interpretation of the results is mired in the uncertainty in experimental parameters and recording noise. However, the magnetic technique is less sensitive to the model parameters [26]. In addition, the absence of any electrical connection between the probe and the tissue makes the probe insensitive to changes in the electrochemical interface between the probe and the sample. Thus, magnetic recordings are not subject to the artifacts that take place in electric recordings during super fusion of media that has altered composition or temperature and tissue motion during recording. During the clinical intraoperative application of the neuromagnetic current probe, the probe and the nerve are immersed in saline, thereby reducing the risk associated with elevating and drying the nerve segment in the air, as required for traditional electric recordings [33,35]. Moreover, the current probe is much less sensitive to stimulus artifacts, which is important in cases in which the proximity of the detector from the stimulating electrode is important [52]. This is due to the geometry of the probe and the low input impedance of the room-temperature amplifier, which allows it to recover faster from the nonlinear behavior produced by an overload, such as that caused by the stimulus, than do high-input-impedance electric amplifiers [9]. However, magnetic technique too has its own boundaries. The spatial resolution of the probe is on the order of $1 \mathrm{~mm}$ when it is placed in proximity to the tissue sample. Therefore, the spatial extent of the propagating action signals must exceed $1 \mathrm{~mm}$ before the configuration of the propagating current pattern can be resolved. However, this resolution requirement can usually be met in most measurements on peripheral nerve or muscle.

\section{Summary}

In this review, the measurements of magnetic field created by biological tissues using the neuromagnetic current probe have been described. This technique was first introduced to the biomedical research community in 1980 . Since then, crushed nerve axons, nerve and muscle bundles, reconstructed nerve bundles, and non-uniform propagation of signals in nerve axons have been broadly investigated. During these studies, this technique showed its superiority over the electric technique. This technique is an invaluable tool in measuring activities of action signals during non-uniform signal propagation and, most importantly, estimating the quality of reconstructed nerves during the recovery period. With the neuromagnetic current probe, we can map the variations in action signals with position by scanning the probe along the tissue preparation, without the risk of damage intrinsic to the traditional intracellular recordings. As it was revealed in several of the studies reviewed here, extracellular electric recordings are more sensitive to physiological parameters and do not have the reproducibility inherent in magnetic recordings. All of these studies have now led to a more comprehensive understanding of the generation of magnetic fields by biological tissues and the neuromagnetic current probe for quantifying some potentially very important clinical aspects, such as trauma and non-uniform action signal propagation in nerve axons.

The Superconducting Quantum Interference Device (SQUID) magnetometers are also widely used to measure magnetic fields created by biological tissues and have their own advantages. In general, the measurements made with neuromagnetic current probes have better spatial resolutions than those measured with the SQUIDs. Another noteworthy advantage that the neuromagnetic current probes has over the SQUIDs is that they can be positioned very close to the living tissues where the magnetic field is strongest leading to a stronger and 
better signal-to-noise ratio. Nevertheless the SQUID magnetometer can be employed to measure magnetic signals noninvasively from living tissues. Although using the SQUID magnetometer may not be efficient for basic research involving isolated nerves or muscles, it is vital when considering the clinical applications of this technique and measuring biological signals that arise from in situ experiments.

\section{Acknowledgements}

The author appreciates the valuable comments made by the reviewers. Some of the figures shown in this manuscript were provided by Profs John P. Wikswo and Bradley J. Roth.

\section{References}

1. Baule G, McFee R (1963) Detection of the magnetic field of the heart. Am Heart J 66: 95-96.

2. Cohen D (1968) Magnetoencephalography: evidence of magnetic fields produced by alpha-rhythm currents. Science 161: 784-786.

3. Cohen D, Givler E (1972) Magnetomyography: magnetic field around the human body produced by skeletal muscles. Appl Phys Lett 21: 114-116.

4. Wikswo JP, van Egeraat JM (1991) Cellular Magnetic Fields: Fundamental and Applied Measurements on Nerve Axons, Peripheral Nerve Bundles, and Skeletal Muscle. J Clin Neurophysiol 8: 170-188.

5. Wikswo JP, Barach JP, Freeman JA (1980) Magnetic Field of a Nerve Impulse: First Measurements. Science 208: 53-55.

6. Barach JP, Freeman JA, Wikswo JP (1980) Experiments on the Magnetic Field of Nerve Action Potentials. J Appl Phys 51: 4532-4538.

7. Wikswo JP (1981) Recent Developments in the Measurement of Magnetic Fields From Isolated Nerves and Muscles. J Appl Phys 52: 2554-2559.

8. Wikswo JP (1982) Improved Instrumentation for Measuring the Magnetic Field of Cellular Action Currents. Rev Sci Instrum 53: 1846-1850.

9. Gielen FLH, Roth BJ, Wikswo, JP (1986) Capabilities of a Torid-Amplifie System for Magnetic Measurement of Current in Biological Tissue. IEEE T Biomed Eng 33: 910-921.

10. Wikswo JP (1983) Cellular Action Currents. Biomagnetism: An Interdisciplinary Approach, Plenum Publishing.

11. Wikswo JP (1985) Magnetic Measurements on Single Nerve Axons and Nerve Bundles. Med Biol Eng Comput 23: 3-6.

12. Wikswo JP (1988) Magnetic Techniques for Evaluating Peripheral Nerve Function. Proceedings of a Special Symposium on Maturing Technologies and Emerging Horizons in Biomedical Engineering, New Orleans, LA, USA.

13. Roth BJ, Wikswo JP (1988) The Magnetic Field of Nerve and Muscle Fibers. Biomagnetism ' $87,6^{\text {th }}$ International Conference on Biomagnetism, Tokyo, Japan.

14. Wikswo JP, Samson PC, Giffard RP (1983) A Low-Noise Low Input Impedance Amplifier for Magnetic Measurements of Nerve Action Currents. IEEE T Biomed Eng 30: 215-221.

15. Wikswo JP, Henry WP, Samson PC, Giffard RP (1985) A Current Probe System for Measuring Cellular Action Currents. Biomagnetism: Applications and Theory, Pergamon Press.

16. Wikswo JP, Barach JP, Gundersen SC, Palmer JO, Freeman JA (1983) Magnetic Measurements of Action Currents in an Isolated Lobster Axon. IL Nuovo Cimento 2D: 512-516

17. Roth BJ, Wikswo JP (1985) The Magnetic Field of a Single Axon: A Comparison of Theory and Experiment. Biophys J 48: 93-109.

18. Roth BJ, Woosley JK, Wikswo JP (1985) An Experimental and Theoretical Analysis. Biomagnetism: Applications and Theory, Pergamon Press.

19. Kitzman GA, Droll PW, lufer EJ (1971) A Theoretical analysis of neurona biogenerated magnetic field. The nervous system and electric currents. Plenum, New York, USA.

20. Scott AC (1977) Neurophysics. Wiley, New York, USA.

21. Swinney KR, Wikswo JP (1980) A Calculation of the Magnetic Field of a Nerve Action Potential. Biophys J 32: 719-732.
22. Plonsey $R$ (1981) Magnetic field resulting from action currents on cylindrical fibers. Med Biol Eng Comput 19: 311-315.

23. Barach JP, Roth BJ, Wikswo JP (1985) Magnetic Measurements of Action Currents in a Single Nerve Axon: A Core-Conductor Model. IEEE T Bio-med Eng 32: 136-140.

24. Woosley JK, Roth BJ, Wikswo JP (1985) The Magnetic Field of a Single Axon: A Volume Conductor Model. Math Biosci 76: 1-36.

25. Wijesinghe RS, Gielen FLH, Wikswo JP (1991) A Model for Compound Action Potentials and Currents in a Nerve Bundle I: The Forward Calculation. Ann Biomed Eng 19: 43-72.

26. Wijesinghe RS, Wikswo JP (1991) A Model for Compound Action Potentials and Currents in a Nerve Bundle II: A Sensitivity Analysis of Model Parameters for the Forward and Inverse Calculations. Ann Biomed Eng 19: 73-96.

27. Wijesinghe RS, Gielen FLH, Wikswo JP (1991) A Model for Compound Action Potentials and Currents in a Nerve Bundle III: A Comparison of the Conduction Velocity Distributions Calculated From Compound Action Currents and Potentials. Ann Biomed Eng 19: 97-121.

28. van Egeraat JM, Stasaski R, Barach JP, Friedman RN, Wikswo JP (1993) The Biomagnetic Signature of a Crushed Axon: A Comparison of Theory and Experiment. Biophys J 64: 1299-1305.

29. van Egeraat JM, Wikswo JP (1993) A Model for Axonal Propagation Incorporating Both Radial and Axial lonic Transport. Biophys J 64: 1287-1298.

30. van Egeraat JM, Friedman RN, Wikswo JP (1990) Magnetic Field of a Single Muscle Fiber: First Measurements and a Core Conductor Model. Biophys $J$ 57: 663-667.

31. Hentz VR, Wikswo JP, Abraham GS (1986) Magnetic Measurement of Nerve Action Currents: A New Intraoperative Recording Technique. Peripheral Nerve and Regeneration 1: 27-36

32. Kline DG, Dejone BR (1968) Evoked potentials to evaluate peripheral nerve injuries. Surgery, Gynecology and Obstetrics 127: 1239-1248.

33. Kline DG, Hackett ER, May PR (1969) Evaluation of nerve injuries by evoked potentials and electromyography. J Neurosurg 31: 128-136.

34. Kline DG, Neulsen FE (1972) Neuroma-in-continuity - its preoperative and postoperative management. Surg Clin of North America 52: 1189.

35. Terzis JK, Dykes RW, Hakstian RW (1976) Electrophysiologic recordings in peripheral nerve surgery - a review. J Hand Surg 1: 52-65.

36. Cummins KL, Perkel DH, Dorfman LJ (1979) Nerve conduction velocity distributions I. Estimation based on the single-fiber and compound action potentials. Electroen Clin Neuro 46: 634-646.

37. Cummins KL, Dorfman LJ, Perkel DH (1979) Nerve conduction velocity distributions II. Estimation based on two compound action potentials. Electroen Clin Neuro 46: 647-658.

38. Wikswo JP, Henry WP, Freidman RN, Kilroy AW, Wijesinghe RS, et al. (1990) Intraoperative recording of the magnetic field of a human nerve. Advances in Biomagnetism, Plenum, New York, USA.

39. Wikswo JP, Abraham GS, Hentz VR (1985) Magnetic assessment of regeneration across a nerve graft. Biomagnetism: Theory and Applications, Pergamon Press, New York, USA.

40. Wijesinghe RS, Roth BJ (2009) Detection of Peripheral Nerve and Skeletal Muscle Action Currents Using Magnetic Resonance Imaging. Ann Biomed Eng 37: 2402-2406

41. Badettini PA, Petridou N, Boduraka J (2005) Direct detection of neurona activity with MRI: fantasy, possibility, or reality? Appl Magn Reson 29: 65-88.

42. Bodurka J, Bandettini PA (2002) Toward direct mapping of neuronal activity: MRI detection of ultraweak transient magnetic field changes. Magnet Reson Med 47: 1052-1058.

43. Cassara AM, Hagberg GE, Bianciardi M, Migliore M, Maraviglia B (2008) Realistic simulations of neuronal activity: A contribution to the debate on direct detection of neuronal currents by MRI. Neurolmage 39: 87-106.

44. Paley MNJ, Chow LS, Whitby EH, Cook GG (2009) Modelling of axonal fields in the optic nerve for direct MR detection studies. Image Vision Comput 27 331-341.

45. Troung T-K, Song AW (2006) Finding neuroelcetric activity under magneticfield 
Citation: Wijesinghe RS (2014) Detection of Magnetic Fields Created by Biological Tissues. J Electr Electron Syst 3: 120. doi:10.4172/23320796.1000120

oscillations (NAMO) with magnetic resonance imaging in vivo. Proc Natl Acad Sci USA 103: 12598-12601

46. Wijesinghe RS, Roth BJ (2009) Effect of Peripheral Nerve Action Currents on Magnetic Resonance Imaging. Proceedings of the $31^{\text {st }}$ Annual International Conference of the IEEE Engineering in Medicine and Biology Society.

47. Wijesinghe RS, Roth BJ (2010) Magnetic Resonance Imaging Technique and Peripheral Nerve Activities. Proceedings of the Indiana Academy of Science 118: $5-8$

48. Kuypers PDL, Gielen FLH, Wai RTJ, Hovius SER, Godschalk M, et al. (1993) A comparison of electric and magnetic compound action signals as quantitative assays of peripheral nerve regeneration. Muscle and Nerve 16: 634-641.

49. Kuypers PDL, van Egeratt JM, Heel MDV, Briemen LJV, Godschalk M, et al. (1998) A magnetic evaluation of peripheral nerve regeneration: I. The discrepancy between magnetic and histologic data from proximal segment. Muscle and Nerve 21: 739-749.

50. Kuypers PDL, van Egeraat JM, Heel MDV, Briemen LJV, Godschalk M, et al. (1998) A magnetic evaluation of peripheral nerve regeneration: II. The signa amplitude in the distal segment in relation to functional recovery. Muscle and Nerve 21: 750-755

51. Sepulveda NG, Wikswo JP (1987) Electric and magnetic fields from twodimensional anisotropic bisyncytia. Biophys J 51: 557-5568.

52. Gielen FLH, Friedman RN, Wikswo JP (1991) In vivo Magnetic and Electric Recordings from Nerve Bundles and Single Motor Units in Mammalian Skeletal Muscle. J Gen Physiol 98: 1043-1061.

53. Wikswo JP (1988) Magnetic Techniques for Evaluating Peripheral Nerve Function. Proceedings of the $10^{\text {th }}$ Annual EMBS Conference, New Orleans, LA USA.

This article was originally published in a special issue, Electromagnetic Wave

Theory handled by Editor(s). Dr. Cheng-Wei Qiu, National University of Sin-

gapore, Singapore. 